\title{
Resultados de un programa educativo con refuerzo audiovisual durante la sesión, en pacientes en hemodiálisis
}

\author{
María Auxiliadora Santana Díez, Mª Rosario Aguirremota Corbera, María del Pilar Lodeiro Herraiz, Marta \\ González Vélez, Sonia Castro Pereira, Julene Escudero Argaluza
}

Hospital Universitario Cruces. Barakaldo. Bizkaia. España

\begin{abstract}
Resumen
Los medios audiovisuales son imprescindibles para reforzar la información del paciente. El objetivo del trabajo es describir la utilidad del uso de presentaciones educativas para los pacientes en hemodiálisis y los medios audiovisuales disponibles en la unidad.
\end{abstract}

Se elaboraron tres presentaciones educativas en $\mathrm{Mi}$ crosoft Office Power Point y se pasaron una serie de cuestionarios con 13 preguntas que hacían referencia a aspectos fundamentales del cuidado tratados en las presentaciones, antes y después de éstas.

Se realizó un análisis estadístico descriptivo con la distribución de frecuencias y porcentajes de las variables categóricas y con la media y la desviación típica de las variables cuantitativas. Para comprobar los resultados de las respuestas antes y después de las presentaciones/micropíldoras se ha utilizado la prueba de McNemar para dos muestras relacionadas con un nivel de significación estadística de $p<0,05$.

El estudio consta de 45 pacientes, $73,3 \%$ varones con una edad media de 63,82 años. Se encontraron resultados estadísticamente significativos con $\mathrm{p}=0,031$ y $p=0,004$, referentes a cuestiones sobre la desinfección de la fístula y el aporte proteico.

El presente estudio ha servido para demostrar que la herramienta ha abierto nuevas posibilidades de formación a los pacientes.

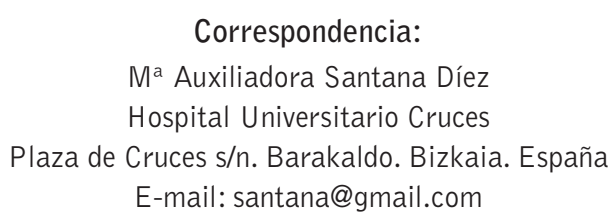

PALABRAS CLAVE: hemodiálisis; información; medios audiovisuales.

Results of an educational program with audio-visual reinforcement during the session in hemodialysis patients

\begin{abstract}
Audiovisual media are essential to reinforce patient information. The objective of this study is to describe the usefulness of educational presentations for hemodialysis patients and available audiovisual media in the unit.
\end{abstract}

Three educational presentations were developed in Microsoft Office Power Point and questionnaires were used with 13 questions regarding fundamental aspects of care approached in the presentations, before and after these presentations.

A descriptive statistical analysis was performed with the distribution of frequencies and percentages of the categorical variables and with the mean and standard deviation of the quantitative variables. To test the results of the responses before and after the presentations, the McNemar's test was used for two related samples, with a statistical significance level of $\mathrm{p}<0.05$.

The study included 45 patients, $73.3 \%$ males with a mean age of 63.82 years. Statistically significant results were found with $p=0.031$ and $p=0.004$, concerning questions about fistula disinfection and protein intake. 
The present study has served to demonstrate that the tool has opened new training possibilities for patients.

KEYWORDS: hemodialysis; information; audiovisual media.

\section{Introducción}

Los medios audiovisuales se usan cada vez más en nuestra sociedad resultando un método de enseñanza muy eficaz. En las salas de hemodiálisis (HD) nos encontramos con demasiada frecuencia pacientes que habiendo sido informados por diferentes profesionales, sorprenden con cuestiones y dudas que se pensaban superadas. Hay diferentes factores que afectan a ésta pérdida de información: al tratarse de una enfermedad crónica, el tiempo hace que poco a poco la olviden, la edad cada vez más avanzada de la población también juega un papel determinante a la hora de ésta pérdida y los casos que comienzan HD de manera urgente sin pasar el periodo previo de consultas ${ }^{1}$.

Se realizó una búsqueda bibliográfica sobre métodos de enseñanza para pacientes en $\mathrm{HD}^{2,3}$, que hicieran referencia a enseñanza a través de medios visuales y enseñanza grupal, el nivel de conocimientos de los pacientes en $\mathrm{HD}^{4}$ y trabajo con medios audiovisuales. Algunos trabajos apoyan la efectividad del apoyo audiovisual y la enseñanza en grupo ${ }^{5,6}$, evaluando la efectividad con cuestionarios pre y post intervención ${ }^{6}$. También se ha publicado la utilidad de las aplicaciones móviles para pacientes en hemodiálisis ${ }^{7}$.

En nuestra unidad desde hace unos años trabajamos en una consulta de enfermería de enfermedad renal crónica avanzada (ERCA) los aspectos más importantes de los cuidados del paciente con patología renal. Elaboramos una guía en papel específica para el paciente en HD que entregamos como apoyo a lo trabajado y respondemos todas las dudas que nos plantean los pacientes. No obstante, consideramos que hace falta información reiterada para los que olvidan, para los que por algún motivo no la tienen o para los que piensan que lo están haciendo bien y tienen algún aspecto mal aprendido.

Como servir la información de una manera útil, lúdica y sobre todo eficaz era una cuestión a resolver. La queja más recurrente en los pacientes que acuden a un centro para realizar HD es la cantidad de horas que invierten en su tratamiento, por eso citarlos fuera de ese tiempo para brindar la información nos pareció que no era una buena idea. Proporcionarla a todos a la vez, de una manera cómoda, en la sala, utilizando los medios audiovisuales que tenemos, una vez comenzada la sesión de HD y antes de que cada uno realice la actividad que quiera (comer algo, ver la televisión, escuchar la radio, leer un libro o simplemente dormir) podía ser más eficaz.

Tenía que ser una intervención no muy larga para mantener su interés teniendo en cuenta la elevada media de edad de los pacientes.

Nuestro principal objetivo era saber si la herramienta audiovisual que hemos utilizado serviría para aumentar la información de los pacientes y sería efectiva la enseñanza grupal.

\section{Material y Método}

Se trata de un estudio analítico, longitudinal, cuasi-experimental y prospectivo. Abarcó desde principios de marzo a mediados de mayo del 2015.

La muestra fue el total de la población en HD y se excluyó solamente a las personas que no quisieron participar 0 aquellas que no podían seguir las explicaciones por problema de idioma o por deterioro cognitivo. También se eliminaron aquellos que no pudieron realizar la segunda encuesta por ingreso, trasplante o defunción.

Se prepararon tres presentaciones Microsoft Office en formato Power Point en su versión 2007:

- "Enfermedad renal crónica", con 19 diapositivas y una duración de aproximadamente 5 minutos.

- "Cómo leer el etiquetado de los alimentos", con 16 diapositivas y una duración de 5 minutos y medio.

- "Cuidados de la fístula arteriovenosa interna", con 12 diapositivas y una duración de 2 minutos y medio.

El trabajo que íbamos a realizar se presentó personalmente a los pacientes mediante una hoja informativa (Anexo 1) la primera semana de marzo del 2015.

Contestaron un cuestionario de elaboración propia (Anexo 2) que constaba de dos partes. En primer lugar se recogían datos personales para conocer las ca- 
racterísticas de nuestra población (edad, sexo, tiempo en HD y si buscan información sobre la enfermedad renal crónica en publicaciones o internet). A continuación tenían que responder a trece preguntas sobre los cuidados en la enfermedad renal crónica, que se iban a tratar posteriormente en las presentaciones.

Se realizó en dos ocasiones, previa a la intervención y después de ésta, para evaluar su eficacia.

Las presentaciones las visualizaron entre marzo y abril del 2015, cada presentación, que denominamos "micropíldora" en una semana distinta, los lunes y martes, para abarcar todos los turnos. Al terminar volvimos a repetir la secuencia para que así vieran cada una en dos ocasiones.

Se recogieron consentimientos informados explicándoles el proceso y garantizando la confidencialidad de las personas participantes en el estudio, así como la posibilidad de abandonarlo cuando quisieran.
En ocho preguntas más el porcentaje de respuestas acertadas en la post-intervención mejoró aunque no era estadísticamente significativo (Tabla $\mathbf{1}$ ).
Tabla 1. Resultados.

\begin{tabular}{|llll}
\hline & $\begin{array}{l}\text { Pre-intervención } \\
\text { Respuestas correctas (\%) }\end{array}$ & $\begin{array}{l}\text { Post-intervención } \\
\text { Respuestas correctas (\%) }\end{array}$ & p-valor \\
\hline Pregunta 1 & $38(84.44 \%)$ & $44(97.78 \%)$ & 0.031 \\
\hline Pregunta 2 & $25(55.56 \%)$ & $30(66.67 \%)$ & 0.302 \\
\hline Pregunta 3 & $19(42.22 \%)$ & $32(71.11 \%)$ & 0.004 \\
\hline Pregunta 4 & $32(71.11 \%)$ & $30(66.67 \%)$ & 0.774 \\
\hline Pregunta 5 & $40(88.89 \%)$ & $41(91.11 \%)$ & 0.999 \\
\hline Pregunta 6 & $37(82.22 \%)$ & $39(86.67 \%)$ & 0.687 \\
\hline Pregunta 7 & $38(84.44 \%)$ & $38(84.44 \%)$ & 0.999 \\
\hline Pregunta 8 & $43(95.56 \%)$ & $42(93.33 \%)$ & 0.999 \\
\hline Pregunta 9 & $38(84.44 \%)$ & $40(88.89 \%)$ & 0.687 \\
\hline Pregunta 10 & $33(73.33 \%)$ & $34(75.56 \%)$ & 0.999 \\
\hline Pregunta 11 & $43(95.56 \%)$ & $44(97.78 \%)$ & 0.999 \\
\hline Pregunta 12 & $24(53.33 \%)$ & $27(60 \%)$ & 0.648 \\
\hline Pregunta 13 & $37(82.22 \%)$ & $40(88.89 \%)$ & 0.549 \\
\hline
\end{tabular}

Se ha realizado un análisis descriptivo con la distribución de frecuencias y porcentajes de las variables categóricas y con la media y la desviación típica de las variables cuantitativas. Para la comparación de los resultados de las respuestas de la encuesta pre-intervención y post-intervención se ha utilizado la prueba de McNemar para dos muestras relacionadas con un nivel de significación estadística de $p<0,05$. Los datos fueron analizados por el software estadístico SPSS versión 23.0.

\section{Resultados}

Nuestro estudio constó finalmente con una población de 45 individuos, en la que el $73,3 \%$ son varones, con una edad media de 63,82 años (D.E. 16,21), de éstos buscan información en publicaciones 0 internet el $44 \%$ y llevan más de 40 meses en HD el 46,7\%.

Encontramos respuesta mejorada, estadísticamente significativa en dos preguntas. En la 1 que hace referencia a la higiene y desinfección de la fístula antes de realizar la $H D$, con una $p=0,031$, pasando el porcentaje de un $84,44 \%$ a un $97,78 \%$ y en la 3 , sobre el control de las proteínas que se ingieren, con una $p=0,004$, pasando el porcentaje de un $42,22 \%$ a un $71,11 \%$.
La pregunta que tuvo un resultado bajo en la preintervención y empeoró post-intervención fue la número 4 , referente a como disminuir el potasio en las comidas.

Analizando los resultados por edad, en menores de 65 años sí se observa una mejora importante en las preguntas 1 y 2 , aunque no son significativas $(p=0,063$ y $p=0,070$ ). Por el contrario, en los mayores de 65 años, en la pregunta 3 sí que hay una mejora significativa $(p=0,039)$ (Tabla 2).

\section{Discusión y conclusiones}

El estudio ha servido para probar la utilidad de una herramienta a nuestro alcance para informar a los pacientes que acuden a HD. En la mayoría de los ítems del cuestionario hemos visto una mejoría en las respuestas y en dos de ellas esta mejoría era estadísticamente significativa. En general, la mejora fue mayor en el grupo de edad más joven, pero no muy diferente al de la población mayor que era una de nuestras preocupaciones al plantear este estudio.

Hemos encontrado dificultades a la hora de rellenar los cuestionarios, ya que por las características de nuestra población, a los pacientes les supuso un es- 
Tabla 2. Resultados.

\begin{tabular}{|c|c|c|c|c|c|c|}
\hline & \multicolumn{3}{|c|}{ Edad $<65(n=22)$} & \multicolumn{3}{|c|}{ Edad $>=65(n=23)$} \\
\hline & Pre-inte. & Post-inte. & $\mathbf{p}$ & Pre-inte. & Post-inte. & $\mathbf{p}$ \\
\hline Pregunta 1 & $17(77.27 \%)$ & $22(100 \%)$ & 0.063 & $21(91.3 \%)$ & $22(95.65 \%)$ & 0.999 \\
\hline Pregunta 2 & $13(59.09 \%)$ & $19(86.36 \%)$ & 0.070 & $12(52.17 \%)$ & $11(47.82 \%)$ & 0.999 \\
\hline Pregunta 3 & $12(54.54 \%)$ & $17(77.27 \%)$ & 0.125 & $7(30.043 \%)$ & $15(65.22 \%)$ & 0.039 \\
\hline Pregunta 4 & $17(77.27 \%)$ & $16(72.72 \%)$ & 0.999 & $15(65.22 \%)$ & $14(60.87 \%)$ & 0.999 \\
\hline Pregunta 5 & $19(86.36 \%)$ & $18(81.81 \%)$ & 0.999 & $21(91.3 \%)$ & $23(100 \%)$ & 0.500 \\
\hline Pregunta 6 & $20(90.91 \%)$ & $20(90.91 \%)$ & 0.999 & $17(73.91 \%)$ & $19(82.61 \%)$ & 0.625 \\
\hline Pregunta 7 & $21(95.45 \%)$ & $22(100 \%)$ & 0.999 & $17(73.91 \%)$ & $16(69.57 \%)$ & 0.999 \\
\hline Pregunta 8 & $22(100 \%)$ & $22(100 \%)$ & - & $21(91.3 \%)$ & $20(86.96 \%)$ & 0.999 \\
\hline Pregunta 9 & $22(100 \%)$ & $22(100 \%)$ & - & $16(69.57 \%)$ & $18(78.26 \%)$ & 0.687 \\
\hline Pregunta 10 & $16(72.72 \%)$ & $17(77.27 \%)$ & 0.999 & $17(73.91 \%)$ & $17(73.91 \%)$ & 0.999 \\
\hline Pregunta 11 & $21(95.45 \%)$ & $22(100 \%)$ & 0.999 & $22(95.65 \%)$ & $22(95.65 \%)$ & 0.999 \\
\hline Pregunta 12 & $10(45.45 \%)$ & $12(54.54 \%)$ & 0.727 & $14(60.87 \%)$ & $15(65.22 \%)$ & 0.999 \\
\hline Pregunta 13 & $20(90.91 \%)$ & $21(95.45 \%)$ & 0.999 & $14(60.87 \%)$ & $21(91.3 \%)$ & 0.727 \\
\hline
\end{tabular}

fuerzo añadido tener que responderlos en dos ocasiones en un lapso corto de tiempo.

Las primeras veces que pasamos las presentaciones/ micropíldoras, preparar el soporte informático y dotar a todos los pacientes de los medios para que pudieran seguir la información, fue un poco complicado para que participaran al mismo tiempo, pero con cada sesión, la preparación fue resultando más sencilla.

Las micropíldoras que fueron analizadas en este estudio aportaban una información muy básica, esto hizo que los resultados en la encuesta pre-intervención fueran en general buenos, lo que dejaba poco margen para que los resultados post-intervención fueran estadísticamente significativos.

Este estudio reafirma lo que otros autores demostraron con sus trabajos 2,3 , el empoderamiento que tiene un paciente cuando recibe información y con ello la eficacia que puede aportar a sus autocuidados.

Hoy en día sabemos que la persona que tiene una enfermedad, sobre todo si ésta es crónica, debe ser gestora de su salud y para ello necesita estar informada, así su tratamiento será más eficaz y la adherencia más elevada.

Gracias a las nuevas tecnologías tenemos más que nunca al alcance de nuestra mano una gran cantidad de información, pero sabemos que no siempre es correcta 0 fácil de interpretar. Por otra parte, la población en HD es cada vez más añosa y no siempre puede acceder a ella y además hay autores ${ }^{8}$ que reflejan que el personal sanitario es el referente principal a la hora de buscar información.

Debemos buscar métodos innovadores ${ }^{7}$, que atraigan su atención, les resulten didácticos, útiles y no requieran un tiempo adicional ${ }^{5}$ al que ya les supone su tratamiento.

Analizando la pregunta 4, relativa a cómo disminuir el potasio en la comida, vimos que no estaba correctamente expresada dando lugar a equívocos. Esto nos ha servido para ser más cuidadosas en la elaboración de posteriores micropíldoras. En ellas hemos intentado que la redacción sea más clara para que el mensaje se entienda correctamente.

A raíz de este estudio, los propios pacientes nos han sugerido temas que les interesan y nos han dado pie a la creación de nuevas micropíldoras. Hemos realizado una nueva sobre los cuidados del catéter venoso central para HD y estamos elaborando otra sobre el ejercicio en HD. Respetando el mismo formato y duración ya que vemos que resulta eficaz.

Es difícil encontrar publicaciones que analicen el impacto de usar nuevos métodos de enseñanza en los pacientes de HD. Es por ello, que este trabajo se enmarca en un tema de investigación de gran interés en la actua- 
lidad, abriendo camino a futuras investigaciones y a la elaboración de posteriores micropíldoras más completas e incluso con diferentes recursos didácticos.

\section{Recibido: 12 noviembre 2016 \\ Revisado: 15 enero 2017 \\ Modificado: 21 febrero 2017 \\ Aceptado: 5 marzo 2017}

\section{Bibliografía}

1. Burgos $E$, Meléndez MA, Meza $E$, Agramon $K G$, Pereyra MC, Martínez NL. Impacto de una intervención dirigida a incrementar el conocimiento de la enfermedad renal, sobre el inicio oportuno de la terapia sustitutiva. Rev Soc Esp Enferm Nefrol. 2011; 14(4):236/241.

2. Lechuga MJ, Rodríguez $A$, Vázquez, EM. Aconsejando \& Educando. Rev Soc Esp Enferm Nefrol.2013; 16 Suppl (1):156/217.

3. Aguilera AI, Prieto M, González L, Abad B, Martínez $E$, Robles I, Gutiérrez $E$, Calleja $A$, de Boso P. Una estrategia poco utilizada en el cuidado de pacientes con enfermedad renal crónica: la educación en grupo y multidisciplinar de pacientes y sus familiares. Rev Soc Esp Enferm Nefrol.2012; 15(1):14/21.
4. Gómez AS, Martín M, Barbero A. Nivel de conocimientos de los pacientes en hemodiálisis. Rev Soc Esp Enferm Nefrol. 2014; 17 Suppl (1): 116/140.

5. Sáez L, Meizoso A, Pérez MJ, Valiño C. Apoyo educativo audiovisual para el paciente en diálisis. Comunicación oral del XXXVI Congreso Nacional de Enfermería Nefrológica. Sevilla. 2011.

6. Torres J, Velasco P, Ila A. Intervención enseñanza a grupo en una unidad de hemodiálisis. Rev Soc Esp Enferm Nefrol.2012; 15 Suppl. (1): 163/180.

7. Casals G, Fenollar M, Morera A, Galceran JM. Impacto de una aplicación (APP) para el enfermo renal. Enferm Nefrol. 2015; 18 Suppl (1): 9/16.

8. González R, Gijón MT, Escudero MJ, Prieto MA, March JC, Ruiz A. Perspectivas de la ciudadanía sobre necesidades y expectativas de información sanitaria. Rev Calidad Asistencial. 2008; 23(3):101-8.

\section{Anexo 1. Guión presentación del estudio a los pacientes}

El personal de enfermería de esta unidad estamos realizando una serie de videos informativos, alguno de los cuales ya están elaborados y los vamos a pasar por la televisión. Son referentes a la enfermedad renal y su tratamiento, duran aproximadamente de 2 a 3 minutos. Vamos a pasar unas encuestas antes y después de los videos, las cuales son anónimas y voluntarias.

Para poder realizarlo os vamos a entregar una hoja de consentimiento informado voluntaria, con el fin de poder utilizar los datos obtenidos en la encuesta y realizar estudios futuros, encaminados a mejorar los conocimientos sobre cuidados en pacientes en hemodiálisis.

Pensamos que os puede ser de una gran utilidad para afianzar lo que ya sabéis, aprender otras nuevas y resolver las dudas que os puedan surgir.

La información la recibiréis durante la diálisis sin influir en los tiempos de entrada y salida, no supondrá ninguna prueba analítica ni médica, solo vuestra atención. 


\section{Anexo 2. Cuestionario pre y post-intervención}

Edad $\square$ Hombre $\square$ Mujer $\square$ Tiempo en diálisis: Años $\square$ Meses $\square$

¿Habitualmente busca información sobre la Insuficiencia Renal Crónica a través de internet, libros, publicaciones u otros medios?

$\mathrm{Si} \square$ No

1) ¿Debemos lavar con agua y jabón y después aplicar un desinfectante en la fistula antes de entrar en hemodiálisis? Si $\square$ No $\square$ No es obligatorio $\square$

2) ¿Cuánto tiempo debo estar comprimiendo la fistula como mínimo para que deje de sangrar a la salida de Hemodiálisis?

$1 \mathrm{~min} . \square 3 \mathrm{~min} . \square 5 \mathrm{~min}$.

3) Para controlar las proteínas que comemos conviene ...

Comer y cenar proteínas $\square$ Evitar los $2^{\circ}$ platos en la cena

Las proteínas se pueden comer libremente

4) ¿Cómo puedo reducir el Potasio en las comidas?

Pongo a remojo el día anterior la legumbre $\square$ Retiro el agua a media cocción $\square$ Ambas cosas

5) ¿Qué se recomienda en todas las fases de la enfermedad renal?

Consumir productos frescos $\square$ Consumir embutidos $\square$ Consumir productos enlatados

6) ¿Qué se recomienda utilizar para dar sabor a las comidas?

Sal $\square$ Pastillas de caldo concentrado $\square$ Especias (ajo, perejil...)

7) En fase avanzada de la enfermedad el exceso de qué sustancias pueden suponer un serio riesgo para la función del corazón y la circulación sanguínea:

Fósforo y potasio $\square$ Vitaminas y minerales $\square$ Hierro y Magnesio

8) ¿Qué alimento de los siguientes debe evitar en su dieta?

Arroz $\square$ Manzana $\square$ Frutos secos $\square$

9) ¿Qué alimento de los siguientes puede comer libremente?
Chocolate
Tomate
Pepino

10) ¿Qué debe evitar en el brazo de la fístula?

Reloj $\square$ Coger objetos pesados con ese brazo $\square$ Ambas respuestas

11) Ante un dolor brusco en la fístula cómo debe actuar...

Esperar a la próxima diálisis $\square$ Acudir urgentemente al hospital $\square$ Esperar a que ceda

12) ¿Qué riesgos se corre con el consumo excesivo de sal?

Insuficiencia cardíaca $\square$ Hipertensión arterial $\square$ Ambas respuestas

13) Sobre 100 gr. de producto, cuál de los que citamos tiene más cantidad de sal
Lechuga
Arroz
Pan 